\title{
Erratum to: Measurement of Moisture Profiles in Pasta During Rehydration Based on Image Processing
}

\author{
Takenobu Ogawa $\cdot$ Shuji Adachi
}

Published online: 17 August 2014

(C) Springer Science+Business Media New York 2014

\section{Erratum to: Food Bioprocess Technol. (2014) 7:1465-1471 DOI 10.1007/s11947-013-1156-y}

We have recently become aware of two mistakes in our published paper Food Bioprocess Technol. (2014) 7:1465-1471 which should be corrected. However, these corrections do not alter the major results, discussion, and conclusions in the paper.

The first mistake is the spatial resolution of the proposed method. The spatial resolution in the original text should be corrected from " $1.6 \mu \mathrm{m}$ " to " $3.2 \mu \mathrm{m}$ " (page 1465 , line 7 in the Abstract, page 1468, line 3 from the bottom in the section "Gray-Level Profile", and page 1471, line 6 in the Conclusions). The pixel sizes of the acquired color images were $1.6 \mu \mathrm{m} / \mathrm{pixel}$, as stated in the text (page 1467, line 31, below the section "Proposed Method"). The camera used is equipped with a filter that has a red, green, and blue mosaic pattern, therefore the spatial resolution of the proposed method using the blue images should be $3.2 \mu \mathrm{m}$. Please note that this correction to the spatial resolution does not detract from the novelty of the proposed method, because the values remain lower than those of the pitches of jaggy patterns of about $20 \mu \mathrm{m}$ in the moisture profiles. As discussed in the section "Gray-Level Profile" in the original paper, these jaggy patterns can be ascribed to the nonuniformity of the starch gelatinization.

The second mistake is caused by an error in the computer program for processing the digital images to generate the blue images: unintended processing was done before pixilation. The error affects the series of figures relating to the images (Figs. 2, $3,4,5$, and 7) and the constants and correlation coefficients in the text. The corrections to the computer program have caused

The online version of the original article can be found at http://dx.doi.org/ 10.1007/s11947-013-1156-y.

T. Ogawa $\cdot$ S. Adachi $(\bowtie)$

Division of Food Science and Biotechnology, Graduate School of Agriculture, Kyoto University, Sakyo-ku, Kyoto 606-8502, Japan

e-mail: adachi@kais.kyoto-u.ac.jp

slight differences in Figs. 2, 4, and 7, and the numerical values in the text; there are also minor differences in the patterns in the gray level and moisture profiles in Figs. 3 and 5, respectively. Although the patterns become jaggier, these jaggy patterns do not change the above discussion. The non-uniform gelatinization of starch is more clearly displayed by this correction. The corrections to the numerical values in the text are as follows,

- $\quad$ Page 1469, line 4, below Eq. (8) should be replaced by "The constants $a, b$, and $c$ were determined to best-fit the observed moisture contents to the calculated ones using the Solver of Microsoft Office Excel ${ }^{\circledR} 2010$ and were $5.12 \times 10^{-5}, 2.56 \times 10^{-3}$, and $-2.20 \times 10^{-1}$ for spaghettini, respectively, and $4.30 \times 10^{-5}, 3.24 \times 10^{-3}$, and $-8.08 \times 10^{-2}$ for lasagna, respectively. The correlation coefficient $\left(R^{2}\right)$ was 0.974 at minimum, indicating the accuracy of Eq. (8) and the validity of the proposed method."

- Page 1470, line 4 from the bottom in the section "Accuracy of Measurement" should be replaced by "The RMSE was 0.182 and 0.138 for spaghettini and lasagna, respectively, indicating the high accuracy of the proposed method for moisture content determinations in pasta."

In addition, the authors request correction to a misprint in a legend. This misprint was caused by inaccurate instructions from the authors at the proofreading stage. On page 1468, the legend for Fig. 2 should be replaced by "Fig. 2 Cross-sectional images of the spaghettini rehydrated for $10.2 \mathrm{~min}(\mathbf{A})$ and the lasagna rehydrated for $14.7 \mathrm{~min}$ (B). (a) Original image and (b) digitally processed image. Broken lines show the segments of moisture profiles as an example"

The authors regret the need for corrections and would like to apologize for any inconvenience caused. 

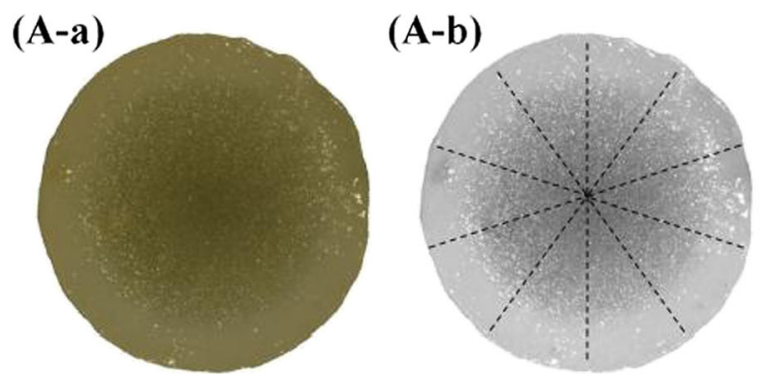

(B-a)

Fig. 2 Cross-sectional images of the spaghettini rehydrated for $10.2 \mathrm{~min}(\mathbf{A})$ and the lasagna rehydrated for $14.7 \mathrm{~min}$ (B). (a) Original image and (b) digitally processed image. Broken lines show the segments of moisture profiles as an example

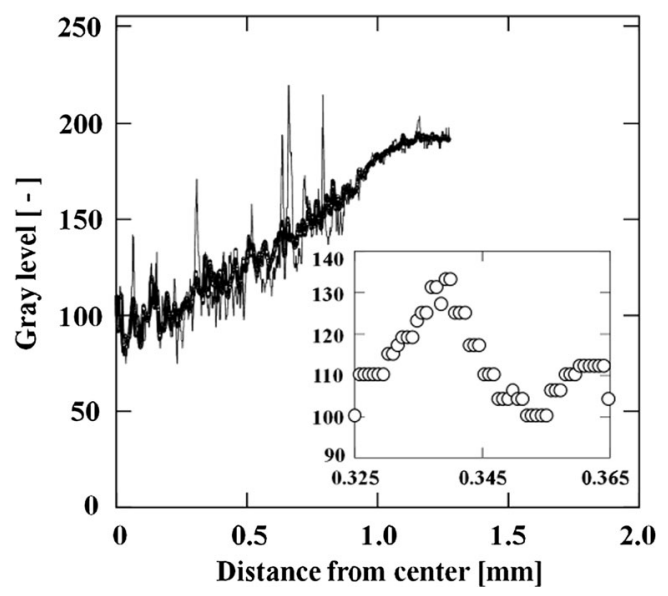

Fig. 3 Gray-level profile of the cross-sectional image of spaghettini rehydrated for $10.2 \mathrm{~min}$. Inset: The extended profile

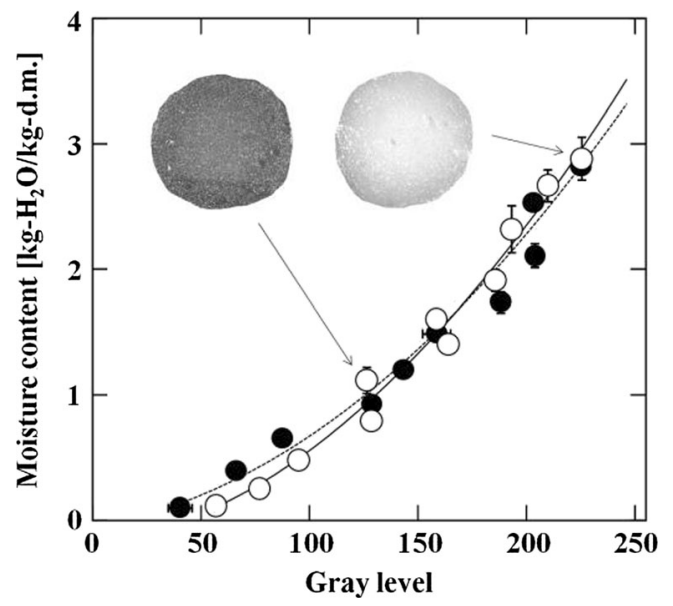

Fig. 4 Relationships between the moisture content and gray level in

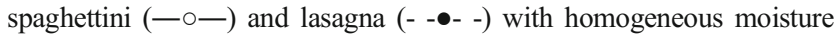
distribution. Bars indicate standard deviation. The pictures in the figure are the digitally processed cross-sectional images obtained from spaghettini with homogenous moisture contents of $1.12 \pm 0.02$ and $2.89 \pm 0.12 \mathrm{~kg}$ $\mathrm{H}_{2} \mathrm{O} / \mathrm{kg}$ d.m., indicated by arrows

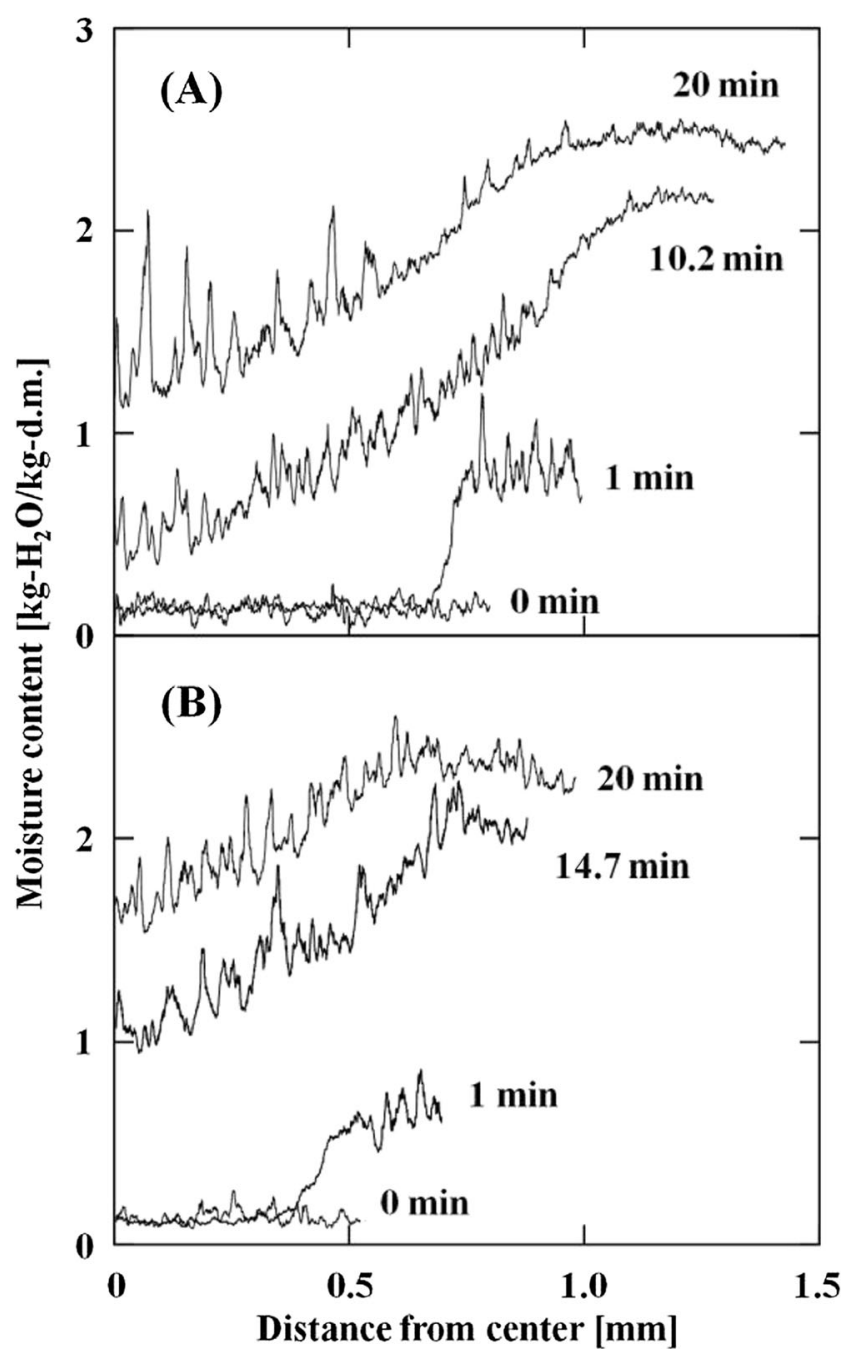

Fig. 5 Moisture profiles of spaghettini (A) rehydrated for 0, 1, 10.2, and $20 \mathrm{~min}$ and lasagna $(\mathbf{B})$ rehydrated for $0,1,14.7$, and $20 \mathrm{~min}$, respectively 


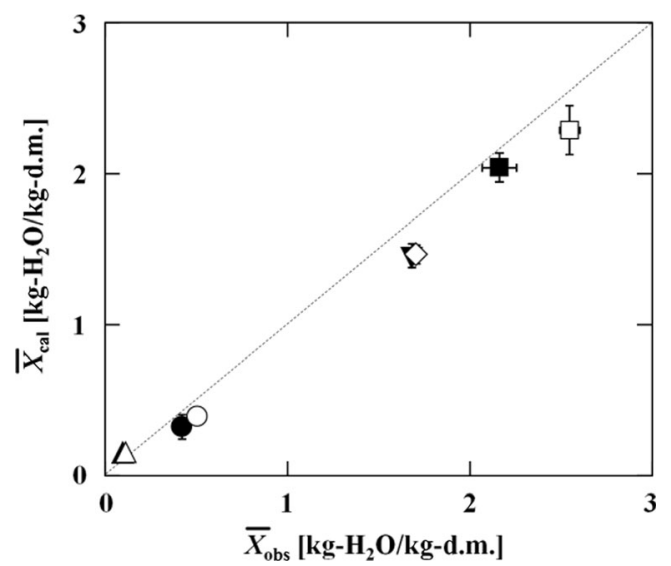

Fig. 7 Correlation between the experimentally observed average moisture content, $\bar{X}_{\mathrm{bs}}$, and the moisture content calculated from the moisture profile, $\bar{X}_{\text {al }}$, in spaghettini rehydrated for 0 min (white up-pointing triangle), $1 \mathrm{~min}$ (white circle), $10.2 \mathrm{~min}$ (white diamond), and $20 \mathrm{~min}$ (white square) and lasagna rehydrated for 0 min (black up-pointing triangle), 1 min (black circle), 14.7 min (black down-pointing triangle), and 20 min (black square). Bars indicating the standard deviation are behind the symbols 